\title{
Efectos de plantas de cobertura sobre la producción de la mandioca en plantío directo
}

\author{
Rosa Nelly Venialgo Chavez. \\ rvenialgo@uni.edu.py \\ Miguel Ángel Bareiro Monzón. \\ miguelbareiro15@gmail.com. \\ Marcos Ariel Pereira Paiva. \\ arielpaiva@gmail.com.
María Gloria Cabrera Romero gloria.cabrera@agr.una.py. \\ Universidad Nacional de Itapúa \\ Itapúa - Paraguay
}

\section{RESUMEN}

Se pretendió obtener información del efecto de plantas de cobertura, sobre la producción de mandioca, en plantío directo, en suelo francoarenoso de Itapúa, Paraguay, de junio 2018 a junio 2021. Fue utilizado un diseño de bloques completamente al azar, con seis tratamientos y cuatro repeticiones. Los tratamientos consistieron en la rotación de plantas de cobertura, $\mathrm{T}_{1}$ (avena negra) + (maíz +mucuna ceniza), $\mathrm{T}_{2}$ (lupino blanco) + (maíz + dolichos), $\mathrm{T}_{3}$ (nabo forrajero $)+($ maíz + Cajanus cajan), $\mathrm{T}_{4}$ (avena negra+ lupino blanco $)+($ maíz + Cajanus cajan $), \mathrm{T}_{5}$ (avena negra + lupino blanco + nabo forrajero $)+($ maíz + Crotalaria juncea $)$ y $\mathrm{T}_{6}($ sin plantas de cobertura). Fueron evaluadas altura de planta, diámetro del tallo, rendimiento de raíces comerciales, rendimiento de raíces no comerciales y rendimiento total de raíces. Los tratamientos con cobertura han presentado mejores crecimientos en altura y diámetro de tallo en relación al testigo. El T5 (avena negra + lupino blanco + nabo forrajero) y (Crotalaria juncea + maíz), presentó mayor rendimiento total de raíces $(14.461 \mathrm{~kg} / \mathrm{ha})$, siendo estadísticamente similar a los demás tratamientos con abonos verdes, a su vez, estas fueron significativamente mayores al $\mathrm{T}_{6}$ (testigo) que tuvo un rendimiento de $1.842 \mathrm{~kg} / \mathrm{ha}$.

Palabras clave: mandioca (Manihot esculenta), planta de cobertura, rotación. 


\title{
Effects of cover plants on cassava production in direct planting
}

\begin{abstract}
It was intended to obtain information on the effect of cover plants, on the production of cassava, in direct planting, in sandy loam soil of Itapúa, Paraguay, from June 2018 to June 2021. A completely randomized block design was used, with six treatments and four repetitions. The treatments consisted of the rotation of cover plants, T1(black oats) $+($ corn + mucuna ash), T2 (white lupine $)+($ corn + dolichos $)$, T3 (forage turnip $)+($ corn + Cajanus cajan), T4 (black oats + white lupine) + (corn + Cajanus cajan), T5 (black oats + white lupine + forage turnip) $+($ corn + Crotalaria juncea) and T6 (without cover plants). Plant height, stem diameter, commercial root yield, non-commercial root yield and total root yield were evaluated. The treatments with coverage have presented better growths in height and stem diameter in relation to the control. The T5 (black oats + white lupine + forage turnip) and (Crotalaria juncea + corn), presented higher total root yield $(14,461 \mathrm{~kg} / \mathrm{ha})$, being statistically similar to the other treatments with green manures, in turn, these were significantly higher than T6 (control) which had a yield of $1,842 \mathrm{~kg} / \mathrm{ha}$.
\end{abstract}

Key words: cassava (Manihot esculenta), cover plant, rotation.

Artículo recibido: 20 diciembre. 2021 Aceptado para publicación: 10 enero 2022 Correspondencia: rvenialgo@uni.edu.py Conflictos de Interés: Ninguna que declarar 


\section{INTRODUCCIÓN}

La mandioca (Manihot esculenta) es una de las mayores fuentes de carbohidratos que consume gran parte de la población paraguaya. Sus raíces, tanto frescas como secas, se emplean en la alimentación humana y animal (Fretes 2010). Prácticamente, se pueden utilizar todas las partes de la planta, esto le confiere características únicas que permiten su total utilización (Armas 2012). Caballero et al. (2019) mencionan que, en Paraguay, la mandioca se cultiva en todos los departamentos de la Región Oriental, cubriendo un área de aproximadamente 182.000 hectáreas. Al respecto, Fretes (2010) refiere que con las técnicas recomendadas es posible alcanzar rendimientos de 25 a 30 t/ha en el primer año, y de 40 a 50 t/ha, en el segundo. Un alto porcentaje del área de cultivo corresponde a fincas de 1 a 20 ha que pertenecen a pequeños y medianos agricultores. Estos, disponen de escasos recursos económicos y utilizan sistemas de producción tradicional con variedades de doble propósito, normalmente criollas, y en siembras asociadas con otros cultivos como el maíz; aunque otros acostumbran a sembrar mandioca en monocultivo. Condiciones de suelo degradado, con la consecuente pérdida de la fertilidad y capacidad productiva, drenaje deficiente y bajo recurso económico del productor, suelen ser limitantes para obtener una buena productividad y que exigen explorar nuevas alternativas en el cultivo de la mandioca. Las coberturas vegetales, producto de la implantación de abonos verdes puros o asociados, que son plantas cultivadas para obtener una cobertura vegetal, una vez desecadas, mecánica o químicamente, se caracterizan por tener varios efectos, entre ellos recuperar, aportar y mejorar las condiciones biológicas, físicas y nutricionales del suelo, promoviendo la conservación del suelo y del agua y con ello la consecuente mejoría de la producción de los cultivos (Jiménez y Añasco 2005). Amabile et al. (1994); Otsubo et al. (2008); Florentín et al. (2001); Araújo et al. 2018) Calegari et al. 1993 y Ojeda et al. 2019 afirman, que la adición regular de residuos de abonos verdes posibilita una mayor acumulación de materia orgánica y de esa manera aporta beneficios para el suelo, proporcionando, consecuentemente, incrementos en la producción agrícola. La respuesta de los cultivos depende, sin embargo, de la interacción de factores como la naturaleza del material incorporado (Turgidez, relación $\mathrm{C} / \mathrm{N}$, contenido de lignina), de las propiedades del suelo, de las características del cultivo principal y del clima. La incorporación de material orgánico al suelo, tiene una contribución fundamental para la 
mandioca, que extrae grandes cantidades de nutrientes (Calegari et al. 1993 y Lal, citado por Amabile et al. 1994). Okigbo, comparando la productividad de raíces de mandioca después de la cobertura de suelo con diversas especies de abonos verdes, encontró aumentos de hasta $80 \%$ en comparación con suelos sin cobertura (Okigbo, citado por Amabile et al. 1994). La relación entre la materia orgánica del suelo y la productividad se atribuye al papel de la materia orgánica en el suministro de nutrientes a las plantas, mejorando la agregación del suelo y, por lo tanto, la capacidad de retención de agua, mejorando el pH del suelo apoyando la actividad biológica y, consecuentemente, dando un mayor rendimiento en comparación con los tratamientos de los que se han eliminado los residuos del cultivo (Matata et al. 2017). Otras experiencias, demuestan que la utilización de cobertura vegetal contribuye a recuperar y aumentar la fertilidad natural de los suelos (Valdivieso 2018). Al respecto, Gilsanz (2008) señala que, en general, la asociación de abonos verdes presenta un mejor desempeño que el empleo de abonos verdes puros en monocultivo. Asimismo, Salazar et al. (2004) refieren que la asociación maíz-frijol incide positivamente y presenta cualidades para su uso dentro de la plantación de mandioca. En consecuencia, se asume que las sucesiones de coberturas vegetales influyen sobre el rendimiento final del cultivo. Es así que, la producción del cultivo de la mandioca sobre rastrojos de plantas de cobertura en asociación, constituye una estrategia que debe ser implementada, considerando que esta tecnología puede presentar importantes efectos sobre el crecimiento y el rendimiento de la mandioca; asimismo, su adopción es de bajo costo económico para los productores.

Por todo lo antes expuesto y, atendiendo a la importancia que representa la mandioca como rubro agrícola en la región, se realizó la investigación con el objetivo de conocer el efecto de las rotaciones con diferentes coberturas vegetales, sobre el rendimiento de la mandioca, bajo siembra directa.

\section{MATERIALES Y MÉTODOS}

El experimento se instaló en la finca experimental de la Facultad de Ciencias Agropecuarias y Forestales, General Artigas, Itapúa, Paraguay, a $138 \mathrm{msnm}$. El clima del país es de tipo subtropical, con temperatura media anual de $22^{\circ} \mathrm{C}$ (Pastén 2009) y precipitaciones anuales de $1.700 \mathrm{~mm}$ (FAO 2015). El suelo de textura franco arenosa corresponde a la clasificación Rhodic Paleudult (López et al. 1995) con 1,64\% de 
materia orgánica; 5,31 de $\mathrm{pH}$ al $\mathrm{H}_{2} \mathrm{O} ; 1,35$ ppm de $\mathrm{P}$ y 20,50 ppm de K. Durante el periodo de ejecución del ensayo, junio del 2018 a junio de 2021, se tuvo una precipitación promedio anual de $1.900 \mathrm{~mm}$. La siembra de las diferentes especies de cobertura de abonos verdes y sus asociaciones fueron realizadas desde junio del año 2018 a mayo del 2020 en la secuencia invierno-verano según tratamiento sobre una rotación anterior del mismo esquema, iniciado en el año 2016. La instalación final del cultivo de mandioca se realizó en agosto del 2020 y la cosecha en el mes de junio del 2021. El diseño experimental utilizado fue de bloques completamente al azar, con seis tratamientos y cuatro repeticiones. El cultivo final (mandioca), se instaló sobre un sistema de rotación previo de cultivos de cobertura o abonos verdes (Tabla 1), constituyendo la combinación y la sucesión de éstos, los tratamientos.

\section{Tabla 1.}

Tratamientos evaluados en el ensayo del efecto de cultivos de coberturas de abonos verdes de invierno y de verano, previo al cultivo de mandioca variedad "Tapojo'a". General Artigas, Itapúa, Paraguay, 2018/21.

\begin{tabular}{|c|c|c|c|c|}
\hline & Tratamientos & & $\begin{array}{c}\text { Especies de invierno } \\
\text { (junio 2018/2019/2020) }\end{array}$ & $\begin{array}{c}\text { Especies de verano } \\
\text { (noviembre 2018/2019) }\end{array}$ \\
\hline $\mathrm{T} 1$ & Ave $+(\text { Maíz+Muc })^{1}$ & & Avena negra & Maíz + Mucuna ceniza \\
\hline $\mathrm{T} 2$ & Lup + (Maíz+Dol $)^{2}$ & & Lupino blanco & Maíz + Dolichos \\
\hline $\mathrm{T} 3$ & $\mathrm{Nab}+(\mathrm{Maiz}+\mathrm{Can})^{3}$ & & Nabo forrajero & Maíz + Canavalia \\
\hline $\mathrm{T} 4$ & $\begin{array}{l}(\text { Ave+Lup) } \\
\text { Maíz+Caj) }\end{array}$ & + & $\begin{array}{l}\text { Avena negra + Lupino } \\
\text { blanco }\end{array}$ & Maíz + Cajanus cajan \\
\hline T5 & $\begin{array}{l}\text { (Ave+Lup+Nab) } \\
(\text { Maíz+Crot })^{5}\end{array}$ & + & $\begin{array}{l}\text { Avena negra + Lupino } \\
\text { blanco + Nabo forrajero }\end{array}$ & Maíz + Crotalaria \\
\hline T6 & Testigo & & Testigo & Testigo \\
\hline
\end{tabular}

Fuente: Elaboración propia a partir de datos relevados de la investigación

1 Ave (Avena strigosa) + Maíz (Zea mays) + Muc (Stizolobium cinereum); ${ }^{2}$ Lup (Lupinus albus) + Maíz (Zea mays) + Dol (Dolichos lab-lab); ${ }^{3} \mathrm{Nab}$ (Raphanus sativus) $+\left(\right.$ Zea mays + Can (Cannavalia ensiformis); ${ }^{4}$ Ave (Avena strigosa + Lup (Lupinus albus) + Maíz (Zea mays) + Caj (Cajanus cajan); ${ }^{5}$ Ave (Avena strigosa) +Lup (Lupinus albus $)+\mathrm{Nab}($ Raphanus sativus $))+$ Maíz (Zea mays) + Crot (Crotalaria juncea)

El cultivo de maíz fue sembrado un mes antes que los abonos verdes de verano según cada tratamiento. El testigo (T6), se mantuvo limpio durante todo el ensayo con carpidas regulares. Posteriormente, al final del ciclo del cultivo de las especies 
mencionadas precedentemente se instaló el cultivo de mandioca variedad Tapojo'a. Cada unidad experimental estuvo separada a $1 \mathrm{~m}$ una de otra, contó con seis hileras de 5 $\mathrm{m}$ de largo y 4,80 m de ancho, totalizando un área de $24 \mathrm{~m}^{2}$; la separación entre plantas fue de $0,70 \mathrm{~m}$ y entre hileras de 0,80 m (17.857 plantas por hectárea). Para la evaluación de rendimiento se utilizó las dos hileras centrales (área útil), de las que se eliminaron 0,70 $\mathrm{m}$, en ambas cabeceras para obviar el efecto de borde, quedando un área neta de $8,64 \mathrm{~m}^{2}$. La separación entre bloques es de 1,00 m.

El trabajo de campo inició en el mes de mayo del año 2018, se realizó la preparación de suelo mediante corpidas y carpidas dejando el suelo libre de malezas para la siembra de los cultivos de cada ciclo, la parcela se instaló sobre una rotación anterior de abonos verdes del mismo esquema, iniciado en el año 2016, totalizando cinco y cuatro ciclos de abonos verdes de invierno y verano, respectivamente.

Tabla 2. Cantidad de semilla, espaciamiento y sistema de siembra de los cultivos de plantas de obertura previo a la instalación del cultivo de la mandioca variedad “Tapojo'a”. General Artigas, Itapúa, Paraguay, 2018/21.

\begin{tabular}{|c|c|c|}
\hline Trat & $\begin{array}{l}\text { Plantas de cobertura de } \\
\text { invierno (kilogramos) }\end{array}$ & $\begin{array}{c}\text { Plantas de cobertura de verano } \\
\text { (separación entre hileras x separación } \\
\text { entre plantas) }\end{array}$ \\
\hline $\mathrm{T} 1$ & $\begin{array}{l}\text { Avena negra: } 80 \quad \mathrm{~kg} / \mathrm{ha}, \\
\text { sembrado al voleo }\end{array}$ & $\begin{array}{l}\text { *Maíz + Mucuna ceniza }(0,50 \text { m x 0,40 m), } \\
\text { sembrados en hoyos }\end{array}$ \\
\hline $\mathrm{T} 2$ & $\begin{array}{l}\text { Lupino blanco: } 60 \mathrm{~kg} / \mathrm{ha} \\
(0,30 \mathrm{~m} \times 0,20 \mathrm{~m}) \text {, sembrados en } \\
\text { hoyos }\end{array}$ & $\begin{array}{l}\text { Maíz + Dolichos }(0,50 \mathrm{~m} \text { x } 0,40 \mathrm{~m}) \text {, } \\
\text { sembrados en hoyos }\end{array}$ \\
\hline $\mathrm{T} 3$ & $\begin{array}{l}\text { Nabo forrajero: } 20 \mathrm{~kg} / \mathrm{ha} \\
\text { sembrado al voleo }\end{array}$ & $\begin{array}{l}\text { Maíz + Canavalia: }(0,50 \mathrm{~m} \text { x } 0,30 \mathrm{~m}) \text {, } \\
\text { sembrados en hoyos }\end{array}$ \\
\hline $\mathrm{T} 4$ & $\begin{array}{l}\text { Avena negra } 40 \mathrm{~kg} / \mathrm{ha} \text {, sembrado } \\
\text { al voleo }+ \text { Lupino blanco } 30 \\
\mathrm{~kg} / \mathrm{ha} \text {, sembrados en hoyos }\end{array}$ & $\begin{array}{l}\text { Maíz + Cajanus cajan }(0,50 \mathrm{~m} \text { x } 0,50 \mathrm{~m}) \text {, } \\
\text { sembrados en hoyos }\end{array}$ \\
\hline T5 & $\begin{array}{l}\text { Avena negra } 26 \mathrm{~kg} / \mathrm{ha} \text {, sembrado } \\
\text { al voleo }+ \text { Lupino blanco } 20 \\
\mathrm{~kg} / \mathrm{ha} \text {, sembrados en hoyos }+ \\
\text { Nabo forrajero } 4 \mathrm{~kg} / \mathrm{ha} \text {, } \\
\text { sembrado al voleo }\end{array}$ & $\begin{array}{l}\text { Maíz + Crotalaria }(0,50 \text { m entre plantas y } \\
48 \text { semillas por metro lineal), sembrados } \\
\text { en surcos }\end{array}$ \\
\hline T6 & $\begin{array}{l}\text { Testigo (sin plantas } \\
\text { cobertura) }\end{array}$ & Testigo (sin plantas de cobertura) \\
\hline
\end{tabular}

* Para el maíz se utilizaron en todos los casos dos semillas por hoyo y una separación de $0,80 \mathrm{~m}$ entre hileras y $0,30 \mathrm{~m}$ entre plantas.

Fuente: Elaboración propia a partir de datos relevados de la investigación 
La siembra de las diferentes especies de plantas de cobertura o abonos verdes de invierno se realizó en junio del año 2018, las cuales, fueron cortados con machete en el mes de septiembre, al inicio de la floración. Posteriormente, se sembró el maíz en octubre del mismo año agrícola. Las especies de abonos verdes de verano, a su vez, fueron sembrados un mes después de la emergencia del maíz, en noviembre del 2018. Los rastrojos provenientes de los abonos verdes de verano, del maíz y cualquier vegetación verde, fueron cortados con un machete seguido de la aplicación de glifosato (2,5 1/ha) para la desecación en mayo del 2019. A partir de la desecación, se sembraron la misma secuencia de plantas de cobertura o abonos verdes de invierno y verano del ciclo anterior en junio de 2019 y noviembre de 2019, respectivamente, conforme puede observarse en la Tabla 1; las mismas fueron cortadas y desecadas, nuevamente, con glifosato en agosto del 2019 y abril del 2020. En mayo de 2020 se repitió la siembra de los abonos verdes de invierno con su posterior corte y desecación en agosto de 2020, previo a la plantación de la mandioca. La parcela testigo se mantuvo limpia, sin vegetación, mantenida con carpidas periódicas durante todo el ciclo. Se eligieron los mejores tallos como rama semilla y se procedió al corte y descarte de la porción basal y la brotación de la parte apical, y ramas deshidratadas, como lo recomiendan Caballero et al. (2019). La plantación se realizó manualmente, en hoyos abiertos a la densidad establecida, en agosto del año 2020, se cosechó en el mes de junio del año 2021. Las labores culturales de eliminación de malezas fueron realizadas con carpidas según necesidad. Fueron evaluadas variables de crecimiento y de producción, tales como: i) Altura de planta $(\mathrm{cm})$. Se midió desde la superficie del suelo hasta el ápice de la planta, a los 10 meses después de la instalación del cultivo. Esta evaluación se realizó en diez plantas representativas al azar del área útil por cada repetición. ii)Diámetro del tallo $(\mathrm{mm})$. Se midió el grosor del tallo al ras del suelo con ayuda de un calibrador vernier de las diez plantas representativas al azar del área útil por cada repetición, al momento de la medición de la altura de la planta (10 meses después de la plantación). iii) Rendimiento de raíces comerciales $(\mathrm{kg} / \mathrm{ha})$. Se clasificaron todas las raíces sin daño mecánico y sin ataque de plagas, ni enfermedades; como raíces comerciales. Se determinó el peso de cada raíz comercial en $\mathrm{kg} / \mathrm{ha}$ de las diez plantas del área útil por repetición iv) Rendimiento de raíces no comerciales (kg/ha). Se clasificaron todas las raíces con daños o ataques de insectos, enfermedades (con pudrición) o con desarrollo 
deficiente (muy finas, pequeñas) como raíces no comerciales. Se determinó el peso de cada raíz no comercial de las diez plantas del área útil en $\mathrm{kg} / \mathrm{ha}$ por repetición en el momento de la cosecha. v) Rendimiento total de raíces ( $\mathrm{kg} / \mathrm{ha}$ ). Se obtuvo de la suma del peso de las raíces comerciales y no comerciales determinadas de las diez plantas del área útil. El resultado se relacionó a $\mathrm{kg} / \mathrm{ha}$ mediante regla de tres simples. Los datos obtenidos se analizaron con el programa estadístico InfoStat ${ }^{\circledR}$ versión 2020 (Di Rienzo et al. 2020). En las variables que resultaron con diferencias significativas, para identificar los tratamientos superiores, se utilizó la prueba de comparación de medias de Tukey al 5\% de probabilidad de error.

\section{RESULTADOS Y DISCUSIÓN}

\section{Parámetros de crecimiento de la mandioca}

En la tabla 3, se presentan los resultados de las variables altura de planta y diámetro del tallo de la mandioca variedad "Tapojo'a", con relación a los diferentes tratamientos de coberturas de suelo con abonos verdes, a los 10 meses después de la plantación.

El análisis de varianza mostró diferencias estadísticas significativas para las variables altura de planta y diámetro del tallo.

Tabla 3. Altura de la planta y diámetro del tallo de la mandioca variedad "Tapojo'a" con relación a los diferentes tratamientos de coberturas de suelo con abonos verdes, a los 10 meses después de la plantación. General Artigas, Itapúa, Paraguay, 2018/21.

\begin{tabular}{|c|c|c|c|c|}
\hline & Tratamientos & $\begin{array}{c}\text { Altura de planta } \\
(\mathrm{cm})\end{array}$ & Diám & $\begin{array}{l}\text { tro del tallo } \\
(\mathrm{mm})\end{array}$ \\
\hline T1 & Ave + (Maíz + Muc) & $133,8 \quad \mathrm{~A}$ & 19,6 & $\mathrm{~A} B$ \\
\hline $\mathrm{T} 2$ & Lup + (Maíz + Dol $)$ & 139,8 A & 22,8 & A \\
\hline $\mathrm{T} 3$ & Nab + (Maíz + Can) & 124,3 A B & 17,9 & $\mathrm{~B} \mathrm{C}$ \\
\hline $\mathrm{T} 4$ & $($ Ave + Lup $)+($ Maíz + Kum $)$ & $134,1 \quad \mathrm{~A}$ & 19,2 & A B \\
\hline T5 & $($ Ave + Lup + Nab $)+($ Maíz + Crot $)$ & $142,1 \quad \mathrm{~A}$ & 21,5 & A B \\
\hline T6 & Testigo & 95,7 & 14,1 & $\mathrm{C}$ \\
\hline Pro & edio & 128,3 & 19,2 & \\
\hline $\mathbf{F} \mathbf{c}$ & ulada & $5,06 * *$ & 10,56 & \\
\hline C.I & $\%)$ & 11,86 & 9,79 & \\
\hline
\end{tabular}

Letras diferentes en la columna indican diferencias significativas al 5\% según Tukey. **: Significativo al $1 \%$.

Como se observa en la Tabla 3, el tratamiento 5, que consistió en la mezcla de abonos verdes de invierno y verano (Ave + Lup + Nab) + (Maíz + Crot), presentó mayor altura 
de planta con 142,1 cm, siendo estadísticamente similares a los tratamientos 2 (Lup) + (Maíz + Dol), 4 (Ave + Lup) + (Maíz + Kum) y 1 (Ave) + (Maíz + Muc).

El tratamiento 6 (Testigo) y el tratamiento $3(\mathrm{Nab})+($ Maíz + Kum), presentaron un promedio de $95,7 \mathrm{~cm}$ y $124,3 \mathrm{~cm}$, respectivamente, siendo los tratamientos con menor altura de planta. El promedio general de altura de planta en este ensayo fue de 128,3 cm. El tratamiento 2 (Lup) + (Maíz + Dol), presentó mayor diámetro de tallo con 22,8 mm, seguido de los tratamientos $5($ Ave + Lup + Nab) + (Maíz + Crot), 1 (Ave) + (Maíz + Muc) y 4 (Ave + Lup) + (Maíz + Kum), siendo éstos estadísticamente iguales entre sí y superiores a los tratamientos $3(\mathrm{Nab})+($ Maíz + Kum) y el tratamiento 6 (Testigo). En el testigo se observó tallos de mandioca con diámetros de 14,1 mm en promedio. El promedio general fue de $19,2 \mathrm{~mm}$.

Los resultados del experimento coinciden con los trabajos de Agbede (2018) y Otsubo et al. (2008) quienes encontraron diferencias significativas en los parámetros de crecimiento de la mandioca, entre la parcela testigo y en los que se cultivaron abonos verdes. El mejor desarrollo vegetativo de la mandioca, reflejado en un mayor crecimiento en altura y diámetro de tallo en los tratamientos con especies de abonos verdes es coherente con lo expresado por Calegari et al. (1993) y Lal, citado por Amabile et al. (1994), en que la incorporación de los abonos verdes en los sistemas productivos de regiones tropicales y subtropicales representa una práctica de respuesta inmediata a la fertilidad y productividad del suelo, pues, contribuye para la protección superficial, al mantenimiento o mejora de las características físicas, químicas y biológicas del suelo por aumento de la materia orgánica, el incremento en la formación de ácidos orgánicos fundamentales en el proceso de solubilización de los minerales del suelo y la mayor disponibilidad de macro y micronutrientes en las camadas superiores del perfil del suelo.

\section{Parámetros de producción de la mandioca}

En la tabla 4, se presentan los resultados de las variables rendimiento de raíces comerciales, rendimiento de raíces no comerciales y rendimiento total de raíces de la mandioca variedad "Tapojo'a", con relación a los diferentes tratamientos de coberturas de suelo con abonos verdes, a los 10 meses después de la plantación. 
Tabla 4. Rendimiento total de raíces $(R T R)$, rendimiento de raíces comerciales (RRC) y rendimiento de raíces no comerciales (RRNC) de la mandioca variedad “Tapojo'a” con relación a los diferentes tratamientos de coberturas de suelo con abonos verdes, a los 10 meses después de la plantación.

\begin{tabular}{|c|c|c|c|}
\hline Tratamientos & RRC (kg/ha) & RRNC (kg/ha & RTR (kg/ha) \\
\hline T1 Ave + (Maíz + Muc) & $8.748,6 \mathrm{~A}$ & $3.381,2$ & $12.129,8 \quad \mathrm{~A}$ \\
\hline T2 Lup + (Maíz + Dol $)$ & $9.447,7 \quad \mathrm{~A}$ & $3.172,7$ & $12.620,4 \quad \mathrm{~A}$ \\
\hline T3 Nab + (Maíz + Can) & $8.274,5 \quad \mathrm{~A}$ & $3.521,4$ & $11.795,9 \mathrm{~A}$ \\
\hline T4 (Ave + Lup $)+($ Maíz + Kum $)$ & $7.928,5 \mathrm{~A}$ & $2.791,1$ & $10.719,6 \mathrm{~A}$ \\
\hline T5 $\quad($ Ave + Lup + Nab $)+($ Maíz + Crot $)$ & $11.727,1 \mathrm{~A}$ & $2.733,9$ & $14.461,1 \quad \mathrm{~A}$ \\
\hline T6 Testigo & 918,3 & 923,7 & 1.842 \\
\hline Promedio & $7,840.8$ & $2.754,0$ & $10.594,8$ \\
\hline F calculada & $9,01 * *$ & 2,07 ns & $9,16 * *$ \\
\hline C.V. $(\%)$ & 31,01 & 47,98 & 27,83 \\
\hline
\end{tabular}

Letras diferentes en la columna indican diferencias significativas al 5\% según Tukey. **: Significativo al $1 \%$ ns: No Significativo

El análisis de varianza demostró que existen diferencias estadísticas significativas entre los tratamientos en donde se utilizaron abonos verdes y el testigo, para las variables rendimiento de raíces comerciales y rendimiento total de raíces (Tabla 4). Como se observa en la misma tabla, el tratamiento 5, que consistió en la mezcla de abonos verdes de invierno y verano $($ Ave + Lup $+\mathrm{Nab})+($ Maíz + Crot $)$, presentó mayor rendimiento de raíces comerciales con $11.727,1 \mathrm{~kg} / \mathrm{ha}$, siendo estadísticamente similares a los tratamientos $2($ Lup $)+($ Maíz + Dol), 1 (Ave) + (Maíz + Muc) 3 (Nab) + (Maíz + Kum) y 4 (Ave + Lup) + (Maíz + Kum). El tratamiento 6 (Testigo) presento un rendimiento de raíces comerciales de $918,3 \mathrm{~kg} / \mathrm{ha}$, siendo estadísticamente diferente a los demás tratamientos, y con marcado menor rendimiento. El promedio general fue de 7.840,8 $\mathrm{kg} / \mathrm{ha}$. Respecto a las raíces no comerciales, no hubo diferencias estadísticas significativas entre los tratamientos donde se utilizaron abonos verdes y el tratamiento 6 (Testigo), el cual resultó con un rendimiento de 923,7 kg/ha. El promedio de rendimiento de raíces no comerciales en el experimento fue de $2.754 \mathrm{~kg} / \mathrm{ha}$. En el rendimiento total de raíces, el tratamiento 5, conformado por la mezcla de abonos (Ave + Lup + Nab) + (Maíz + Crot), arrojó los mayores valores en los atributos de producción de la mandioca, teniendo un rendimiento total de $14.461 \mathrm{~kg} / \mathrm{ha}$ siendo así, 
estadísticamente similares a los demás tratamientos donde se utilizaron abonos verdes, a su vez, éstas fueron significativamente mayores al tratamiento 6 (testigo).

El rendimiento total de raíces no coincide con el resultado que obtuvieron Valenzuela et al. (2020), quienes no obtuvieron diferencias significativas en el rendimiento de raíces con la utilización de C. juncea en el cultivo de mandioca. Sí coinciden, con los resultados de Amabile et al. (1994) quienes obtuvieron mayores rendimientos de raíces en los tratamientos que recibieron la incorporación de C. juncea, misma especie de abono verde utilizada para el tratamiento 5. Los resultados de mayor rendimiento de raíces en las parcelas con abonos verdes en general y el del tratamiento 5 (Ave + Lup+ $\mathrm{Nab})+($ Maíz + Crot) en particular, puede deberse a la mezcla de diferentes especies, que conjugan los beneficios de la sinergia generada entre las especies, por las diferentes características deseables desde el punto de vista del mejoramiento de las propiedades del suelo y la mayor disponibilidad de nutrientes, especialmente, el nitrógeno, uno de los nutrientes más necesarios para un buen crecimiento y producción de la mandioca. La utilización de mezcla de gramíneas, brasicaceas y leguminosas como abono verde, probablemente han generado un equilibrio entre el aporte gradual de nutrientes, considerando las aptitudes de buen reciclador de nitrógeno y potasio de la avena negra, la rápida liberación del nitrógeno por la mineralización del nabo forrajero por su baja relación $\mathrm{C} / \mathrm{N}$ y el importante aporte en nitrógeno fijado y lo mineralizado por el lupino y la Crotalaria, y durante todo el ciclo del cultivo el aporte de nitrógenos por la mineralización del rastrojo del maíz.

Se ha logrado un aumento del $85,1 \%$ en promedio en el rendimiento de la mandioca en aquellas parcelas con cobertura de abonos verdes en general y aumentos de $87,3 \%$ en el rendimiento de la mandioca entre el T5 (Ave + Lup + Nab) + (Maíz + Crot) y la parcela sin cobertura (T6), en absoluta concordancia con lo reportado por Okigbo (Okigbo, citado por Amabile et al. 1994), quien comparando la productividad de raíces de mandioca después de la cobertura de suelo con diversas especies de abonos verdes, encontró aumentos de hasta $80 \%$ en comparación con suelos sin cobertura. Se observó que el coeficiente de variación para rendimiento de raíces ya sea, comerciales y rendimiento total presentan valores de $\mathrm{CV}$ entre $31,01 \%$ y $27,83 \%$, respectivamente, valores muy cercanos con otros experimentos que involucran producción de cultivos en rotación con abonos verdes o asociaciones de cultivos. Comparando estos valores con 
el trabajo de Granato de Andrade et al. (2005), en un experimento de manejo de abono verde en la producción de mandioca en suelo arenosos, el CV fue 38,66 \%. De igual modo, Ferreira De Oliveira (2015) en su experimento de producción de mandioca en función de abono verde, biofertilizante y compuesto orgánico, ha obtenido resultados con valores de CV promedio de 21,5\% para peso de raíces comerciales y 19,84\% para peso total de raíces. Asimismo, comparando con reporte de investigaciones para cultivos de maiz asociado con poroto, Cantero y Pereira (2018) han obtenido valores de CV en torno de $26,39 \%$ para rendimiento de maíz y 30,36 \% para rendimiento de poroto. Cardama-Vazquez (1988) han registrado valores de CV de 70,55 \% para rendimiento de cultivos asociados, de caupí y de 54,01 \% en rendimiento de maíz, promedios de resultados de análisis combinados de rendimientos de tres años. Se asume que, a parte del manejo del sistema productivo semejante a las experiencias mencionadas, otro factor importante del que derivan estos valores de CV se debería a la heterogeneidad intrínseca natural del terreno, al no existir labores de recuperación de suelo anteriores al experimento realizado. Cochran y Cox, citado por Ruiz-Ramírez (2009) sostienen que cuando se tiene en el sitio experimental un gradiente de condiciones no controlables, como en el caso de la pendiente del terreno, podría afectar la respuesta a los tratamientos.

\section{CONCLUSIONES}

Conforme a los resultados obtenidos en la presente investigación y bajo las condiciones en las que se condujo el experimento se concluye que:

El crecimiento y producción de la mandioca fue influenciado positivamente por la utilización y rotación de los abonos verdes de cobertura, tanto los de cultivos de invierno como los de verano. La altura de planta, el diámetro de tallo y la producción de raíces tuberosas fueron superiores en las parcelas cubiertas con abonos verdes que aquellas sin cobertura. La utilización de la combinación de abonos verdes (Avena negra + Lupino blanco + Nabo forrajero y Maíz + Crotalaria juncea) arrojó los mayores valores de crecimiento de las plantas, así como de producción, a pesar de no presentar diferencias con relación a las demás combinaciones de abonos verdes. 


\section{REFERENCIAS BIBLIOGRÁFICAS}

Agbede, T. 2018. Effect of Green Manure Application on Cassava (Manihot esculenta Crantz) Growth, Yield Quantity and Quality in Degraded Alfisols. Pertanika J. Trop. Agric. Sc. 41(4):1.757-1.777.

Amabile, R.F; Correia, J.R; DE Freitas, P.L; Blancaneaux, P; GamalieL, J. 1994. Efeito do manejo de adubos verdes na produção de mandioca (Manihot esculenta Crantz). Pesq. Agropec. 29(8):1.193-1.199.

1994. Efeito do manejo de adubos verdes na produção de mandioca (Manihot esculenta Crantz). Pesq. Agropec. 29(8):1.193-1.199. Fuente original: Lal, R. Soil Surface management in the tropics for intensive land use and high and sustained production. Advanced in Soil Sciences, 5:1-109.

. 1994. Efeito do manejo de adubos verdes na produção de mandioca (Manihot esculenta Crantz). Pesq. Agropec. 29(8):1.193-1.199. Fuente original: Okigbo, BN. Farming systems and soil erosion in west Africa. In: GREENLAND, DJ; Lal, R. Soil conservation and management in the humid tropics. London: J. Wiley, 1977. p- 151-164.

ARMAS, F.M. 2012. Instructivo técnico de la yuca (en línea). Morona Santiago, Ecuador. 30 p. Consultado 09 jul. 2020. Disponible en https://es.slideshare.net/INGPAKOWPN/instructivo-tecnico-del-cultivo-de-layuca-msc-ing-francisco-martin-armas.

Caballero, C.A; Enciso Garay, C.R; Tullo ArguellO, C.C; González Villalba, J.D. 2019. Guía técnica: cultivo de mandioca. San Lorenzo, Paraguay, FCA, UNA. 56 p.

Calegari, A; Mondardo, A; Busilani, E; Wildner, L; Costa, M; Alcantara, P; Miyasaka, S; Amado, T. 1993. Adubação Verde no Sul do Brasil. 2 ed. Rio de Janeiro, Brasil. 346 p.

Cantero, M; Pereira, C. 2018. Determinación del momento de siembra en el cultivo asociado maíz (Zea mays L.) y poroto (Vigna unguiculata W.). Tesis. Gral. Artigas.

Cardama-Vazquez, I.O. 1998. Determinación de la frecuencia de siembra del cultivo asociado Caupi (Vigna unguiculata W.) y maíz (Zea mays L.). Folla Amazonica $1(1-2): 49-64$. 
Di Rienzo, J.A; Casanoves, F; Balzarini, M.G; Gonzalez, L; TabladA, M; Robledo, C.W. 2020. InfoStat: versión 2020. Grupo InfoStat, FCA, Universidad Nacional de Córdoba, Argentina.

Ferreira De Oliveira, J.P. 2016. Produção de mandioca em função da adubação verde, biofertilizante e composto orgánico. Programa de Pós-Graduação em Produção Agrícola. Garanhuns, Brasil. 69 p.

Florentín, M.A; Peñalva, M; Calegari, A; DerpscH, R. 2001. Abonos verdes y rotación de cultivos en siembra directa: pequeñas propiedades. San Lorenzo, Paraguay, MAG-GTZ. 84 p.

Fretes, F. 2010. Mandioca: una opción industrial. Paraguay, USAID. 54 p.

Gilsanz, JC. 2008. Jornada de abonos verdes: jardín de abonos verdes de invierno (en línea). INIA. Uruguay, Programa Nacional de Producción y Sustentabilidad Ambiental, Serie Actividades de Difusión N ${ }^{\circ}$ 546. Consultado 1 oct. 2018. Disponible en http://www.inia.uy/Publicaciones/Documentos\%20compartidos/1842926110809 5122.pdf.

Granato De Andrade, A; Lopes De Almeida, D; María De AquinO, A; De-Poli, H. 2005. Manejo da Adubação Verde para Produção de Mandioca em Solo Arenoso (en línea). Ministério da Agricultura, Pecuaria e Abastecimiento. Consultado 23 sept 2021. Disponible en https://ainfo.cnptia.embrapa.br/digital/bitstream/CNPS-2010/14898/1/circtec322005-adubacao-verde.pdf.

Jiménez, W; Añasco, A. 2005. Cultivos de cobertura y abonos verdes. San José, Costa Rica, CEDECO. 33 p. (Serie Agricultura Orgánica Nº).

López Gorostiaga, O; González Erico, E; De LLamas, P.A; Molinas, A.S; Franco, E.S; García, S; Ríos, E.O. 1995. Estudio de reconocimiento de suelos, capacidad de uso de la tierra y propuesta de ordenamiento territorial preliminar de la Región Oriental del Paraguay. Asunción, Paraguay. 246 p.

Otsubo, A.A; Mercante, F.M; Silva, R.F; BorgeS, C.D. 2008. Sistemas de preparo do solo, plantas de cobertura e produtividade da cultura da mandioca. Pesq. Agropec. Bras. 43(3):327-332. 
Ruíz-RamíreZ, J. 2009. Eficiencia relativa y calidad de los experimentos de fertilización en el cultivo de caña de azúcar (en línea). Terra Latinoamericana. 28(2):149154. Consultado 29 sept 2021. Disponible en http://www.scielo.org.mx/scielo.php?script=sci_arttext\&pid=S0187$\underline{57792010000200006 .}$

Salazar Rosero, M.A; Prager Mosquera, M; AraraT Rojas, J.E. 2004. Evaluación de abonos verdes en el cultivo de yuca Manihot esculenta Krantz en un inceptisol de la zona de ladera del Departamento del Cauca, Colombia. Rev. Fac. Nac. Agron. Medellín 57(2):2.413-2.422.

Valdivieso, MB. 2018. Los abonos verdes o cultivos de cobertura (en línea, blog). Ecuador, Sal. Org. Sost. Consultado 8 oct. 2018. Disponible en saludorganicasostenible.com/abonos-verdes/amp/.

Valenzuela, W; Obrador, J; García, E; Castelán, M; Carrillo, E. 2020. Rendimiento comparativo de la yuca bajo fertilización mineral y abono verde. Rev. Mexicana Ciencias Agrícolas. 11(6):1259-1271. 